Klinichna khirurhiia. 2018 August;85(8):21-25.

DOI: $10.26779 / 2522-1396.2018 .08 .21$

\title{
Чрескожная эндоскопическая гастростомия: показания, техника, осложнения и результаты
}

\section{Т. В. Бухарин ${ }^{1}$, В. А. Яковенко ${ }^{1,2,3}$, Ю. В. Фломин ${ }^{1}$, Н. А. Мендель ${ }^{1,4}$}

${ }^{1}$ Медицинский центр «Универсальная клиника «Обериг», г. Киев,

${ }^{2}$ Научно-практический центр профилактической и клинической медицины Государственного управления делами, г. Киев,

${ }^{3}$ Национальный медицинский университет имени А. А. Богомольца, г. Киев,

${ }^{4}$ Национальная медицинская академия последипломного образования имени П. Л. Шупика, г. Киев

\section{Transcutaneous endoscopic gastrostomy: indications, technique, complications and results}

\author{
T. V. Bukharin' ${ }^{1}$ V. A. Yakovenko ${ }^{1,2,3}$, Yu. V. Flomin ${ }^{1}$, N. A. Mendel1,4 \\ ${ }^{1}$ Medical Centre «Universal Clinic «Oberig», Kyiv, \\ ${ }^{2}$ Scientific-Practical Centre of prophylactic and Clinical Medicine, Kyiv, \\ ${ }^{3}$ Bogomolets National Medical University, Kyiv, \\ ${ }^{4}$ Shupyk National Medical Academy of Postgraduate Education, Kyiv
}

\begin{abstract}
Реферат
Цель. Обобщение данных литературы и собственного опыта выполнения чрескожной эндоскопической гастростомии (ЧЭГ).

Материалы и методы. За период с 2011 по 2018 г. 105 пациентам клиники «Обериг» выполнена ЧЭГ. Проанализированы результаты, показания, противопоказания, методика установки гастростомы, особенности лечения пациентов, осложнения.

Результаты. Выполнение ЧЭГ было технически успешным во всех наблюдениях. Всего отмечено 5 (4,8\%) осложнений (95\% доверительный интервал (Ди) 1,6 - 10,8): несостоятельность гастростомы с перитонитом (1), пневмоперитонеум (1), кровотечение из брюшной стенки (1), инфильтрат мягких тканей передней брюшной стенки (1), миграция бампера гастростомы в стенку желудка (1). В сроки наблюдения 30 дней летальности не было.

Выводы. ЧЭГ - безопасный, доступный миниинвазивный метод, который является выбором у пациентов, требующих длительного энтерального питания или длительной декомпрессии желудка. Необходимы строгое соблюдение всех технических этапов ЧЭГ, показаний и противопоказаний, правильный послеоперационный уход за стомой.

Ключевые слова: чрескожная эндоскопическая гастростомия; показания; противопоказания; осложнения; техника выполнения; лечение пациентов.

\section{Abstract}

Objective. To analyze the literature data, summarized together with own experience of the transcutaneous endoscopic gastrostomy (TEG) performance.

Materials and methods. TEG was conducted in 2011 - 2018 yrs to 105 patients in the «Oberig» Clinic. The results, indications, the procedure conduction technique, peculiarities of the patients' treatment, and complications were analyzed.

Results. The TEG conduction was technically successful in all observations. In total 5 (4.8\%) complications (95\% доверительный интервал (ДИ) 1.6 - 10.8) were noted: insufficiency of the gastrostoma sutures with complication by peritonitis (1), pneumoperitoneum (1), bleeding from abdominal wall (1), infiltrate of the anterior abdominal wall soft tissues (1), a gastrostomy bumper migration into gastric wall (1). A 30-days lethality was absent.

Conclusion. TEG is a secure, feasible miniinvasive procedure, which is indicated in patients, who need durable enteral feeding or prolonged gastric decompression. Strict following of all technical stages of the gastrostomy operation performance, as well as the indications and contraindications rules, a correct postoperative care for stoma are mandatory.

Keywords: transcutaneous endoscopic gastrostomy; indications; contraindications; morbidity; technique of performance; treatment of patients.
\end{abstract}

Чрескожная эндоскопическая гастростомия (ЧЭГ) это метод доступа к пищеварительному каналу пациента с целью проведения долгосрочного энтерального питания или декомпрессии желудка.

Энтеральному питанию отдают предпочтение в сравнении с парентеральным, так как оно более физиологично, в 8 - 10 раз дешевле, не требует строгих стерильных условий, а также снижает риск бактериальной транслокации и бактериемии $[1,2]$. Доступ к пищеварительному каналу пациента для энтерального питания возможен с сохранением анатомической целостности пищеваритель- ного канала (использование назогастральных или назоэнтеральных зондов) или с выполнением специальных хирургических вмешательств - наложения различных свищей (стомии) [3, 4]. Назогастральные, назодуоденальные или назоеюнальные зонды, как правило, используют кратковременно - до 30 сут, так как их длительное использование повышает риск осложнений, таких как пролежни пищевода, трахеопищеводные свищи, кровотечение (по ходу зонда), аспирационная пневмония [5]. Если энтеральное питание необходимо осуществлять длительно (свыше 4 нед), больному необходима установка гастро- 
стомы $[1,3,4]$. ЧЭГ показана пациентам со степенью недоедания от умеренной до тяжелой, которые находятся на зондовом питании в течение 3-4 нед, для обеспечения питательной поддержки с целью удовлетворения метаболических потребностей пациентов с неадекватным пероральным приемом пищи и для декомпрессии желудка [3, 4]. Продолжается обсуждение показаний для ЧЭГ у пациентов с тяжелым диабетом, тяжелой деменцией, а также у лиц в возрасте старше 80 лет [6, 7]. Решение о выполнении ЧЭГ принимают индивидуально с учетом потребностей пациента, основного диагноза и ожидаемой продолжительности жизни. Цель состоит в том, чтобы улучшить не только выживаемость и состояние питания пациента, но и качество жизни, уровень которого не обязательно коррелирует с улучшением питания [8].

Первая ЧЭГ была выполнена в 1980 г. как метод выбора для пациентов, которые требуют долгосрочного энтерального питания [9]. Данная методика стала альтернативой открытой гастростомии [4, 9, 10], так как обеспечивает более простой и безопасный доступ к желудку. Изза малой инвазивности выполнение ЧЭГ в большинстве наблюдений возможно без общей анестезии, что важно для ослабленных, истощенных, коморбидных больных.

В то же время данная техника недостаточно широко распространена в Украине.

Цель исследования: обобщение данных литературы и собственного опыта выполнения ЧЭГ.

\section{материал и методы исследования}

Гастростому устанавливали с использованием «Pull technique». ЧЭГ выполняли натощак (полный голод на протяжении 8 ч до процедуры). За 1 ч до гастростомии вводили профилактическую дозу антибиотиков - внутривенно 1 - 2 г цефазолина (в зависимости от массы тела пациента) согласно рекомендациям N. S. Jafri и соавторов, A. Lipp и G. Lusardi $[11,12]$. Положение пациента при ЧЭГ - лежа на спине. Необходимость седации определяли индивидуально.

Выполняли эзофагогастродуоденоскопию, оценивали состояние слизистой оболочки (наличие язвенных дефектов, эрозий) непосредственно в области наложения гастростомы. Наличие язв и эрозий вне зоны гастростомии не является противопоказанием для ЧЭГ. Как прави- ло, накладывали гастростому в средней трети тела желудка по передней стенке, что соответствовало проекции на коже эпигастральной области слева от белой линии живота. Обязательным условием для выполнения процедуры является наличие эффекта трансиллюминации на кожу передней брюшной стенки в зоне пункции желудка. Для установки гастростомы использовали одноразовые стерильные наборы для ЧЭГ 24Fr компании Boston Scientific (США).

В точке наложения гастростомы применяли местную анестезию, выполняли разрез кожи на 1 - 2 мм длиннее диаметра гастростомической трубки. По разрезу пунктировали желудок иглой-троакаром $(p u c .1, a)$. После извлечения стилета по гильзе в желудок вводили проводник (рuс. 1, б), который захватывали эндоскопической петлей, введенной в желудок через инструментальный канал гастроскопа, и проводник вместе с гастроскопом извлекали наружу через пищевод и ротовую полость. К проводнику, выведенному через рот, крепили гастростомическую трубку, которую при помощи тракции за дистальный конец проводника низводили в желудок, а далее - на переднюю брюшную стенку. Снаружи фиксатором ограничивали свободный ход гастростомической трубки на расстоянии $1-2$ см (с учетом дыхательной экскурсии). После установки гастростомы проводили контрольный эндоскопический осмотр желудка для оценки положения ее бампера, выявления возможного кровотечения (рис. 1, в).

Активные реабилитационные мероприятия с пациентами возобновляли на следующие сутки от момента установки гастростомы. После операции уделяли особое внимание уходу за гастростомой. Обязательно выполняли ежедневную обработку кожной раны раствором антисептика с заменой асептической повязки.

Кормление через стому начинали через сутки с момента ее установки. Многие исследователи, включая метаанализ, изучали безопасность кормления, начатого через от 1 - 6 ч после выполнения ЧЭГ, и пришли к выводу, что кормление через 4 ч после гастростомии безопасно [13, 14]. Кормили пациентов густыми питательными смесями в положении лежа на правом боку и в таком положении их оставляли на 30 мин (первые 7 дней). Объем вводимой смеси за одно кормление не превышал 200 мл. Каждое кормление или введение лекарств заканчивали промы-

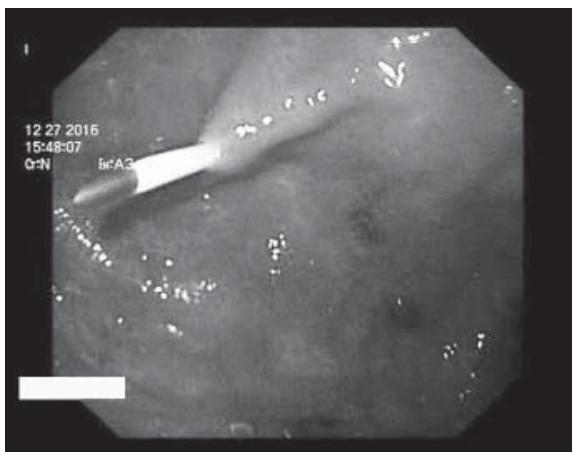

$\boldsymbol{a}$

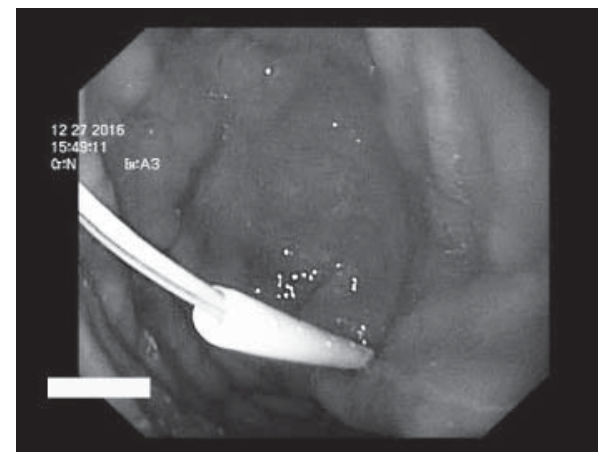

б

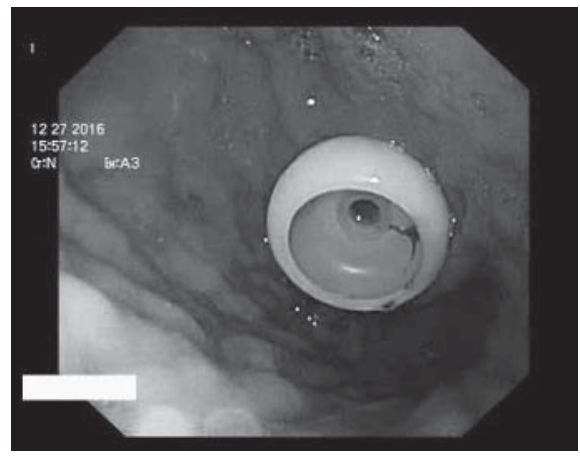

$\boldsymbol{B}$

Puc. 1.

Техника выполнения ЧЭГ:

a - пункция передней стенки желудка; б - проводник в полости желудка; в - балпер гастростомы на передней стенке желудка. 


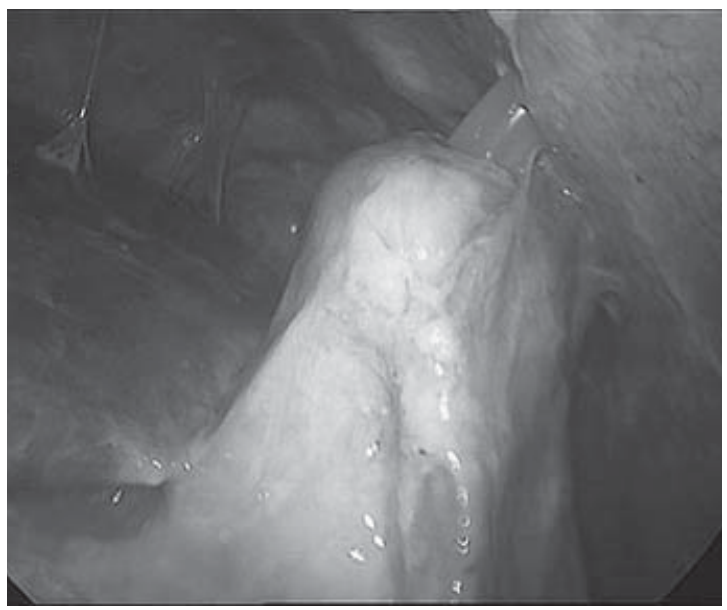

Puc. 2.

Лапароскопия: несостоятельность гастростомы. Видеоролик доступен по адресу bttps://wwwyoutube.com/watch?v=2_BOdmep_o8.

ванием гастростомической трубки чистой водой в объеме 50 мл во избежание ее засорения. Кратность и объем кормления устанавливал врач-диетолог индивидуально.

Весь период, когда у пациента оставалась гастростома, он получал ингибиторы протонной помпы (пантопразол, эзомепразол) в дозе 80 мг в сутки, разделенной на два приема (измельченные и растворенные в воде таблетки вводили через гастростомическую трубку). При необходимости плановую замену гастростомической трубки осуществляли не позднее чем через 6 мес от момента установки или удаляли ее.

В отделении эндоскопии и миниинвазивной хирургии Универсальной клиники «Обериг» за период с 2011 по март 2018 г. выполнено 105 гастростомий, из них 84 первичных и 21 рестомия. Выполнены планово по истечении 6-месячного срока 14 рестомий, по поводу преждевременного удаления гастростомы самим пациентом - 6, в срок 18 мес при развитии бампер-синдрома - 1. у 2 пациентов выполнена лапароскопически ассистированная ЧЭГ (в условиях операционной, под наркозом).

Пациенты были в возрасте от 23 до 92 лет, средний возраст составил 60 лет. Мужчин - 71, женщин - 34. Нуждались в проведении длительного энтерального питания 104 пациента. Гастростомия для декомпрессии желудка в связи с карциноматозом брюшной полости, вызванным аденокарциномой поджелудочной железы, выполнена 1 пациенту. Проходил лечение и реабилитацию в инсультном центре клиники 101 пациент, в стационарном отделении - 4 пациента.

Гастростомия при опухоли левой лобной доли (глиобластоме) выполнена 1 больному, геморрагическом инсульте -27 , ишемическом инсульте - 37, черепно-мозговой травме - 19, огнестрельном ранении головного мозга 2 , боковом амиотрофическом склерозе -1 , неходжкинской В-клеточной лимфоме - 2, энцефалопатии - 10 , внемозговой опухоли (менингиоме) - 2, болезни Паркинсона - 2, аденокарциноме поджелудочной железы с карциноматозом брюшной полости - 1, раке левого легкого со сдавлением нижней трети пищевода -1.

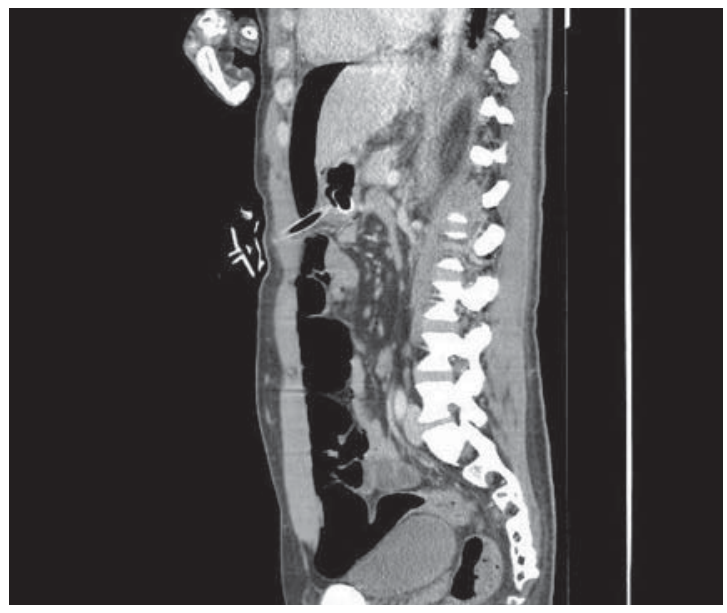

Puc.3.

КТ: диастаз между передней стенкой желудка и передней брюшной стенкой.

Местную анестезию использовали у 94 пациентов, внутривенный наркоз - у 6, наркоз с интубацией трахеи - у 5.

Устанавливали гастростому в кабинете эндоскопии на транспортировочной каталке без перекладывания пациента на стол. Исключением были 5 пациентов, которым ЧЭГ выполнена в условиях операционной под эндотрахеальным наркозом. Время установки гастростомы не превышало 15 мин. Необходимость выполнения лапароскопически ассистированной гастростомии была обусловлена релаксацией купола диафрагмы и, как следствие, отсутствием эффекта трансиллюминации на переднюю брюшную стенку. У этих пациентов желудок пунктировали под контролем лапароскопа.

\section{Результаты}

ЧЭГ удалось выполнить во всех наблюдениях. Сроки нахождения гастростомы составили от 1 до 6 мес, в одном наблюдении - 18 мес, что обусловлено потерей контакта с пациентом, в дальнейшем у него возник бамперсиндром. Шесть пациентов преждевременно спонтанно удалили гастростомические трубки, поворачиваясь в кровати или находясь в состоянии нарушенного сознания. Этим пациентам выполнена рестомия. Данные наблюдения расценивали как осложнения.

Всего возникло 5 (4,8\%) осложнений. В сроки наблюдения 30 дней летальности не зафиксировано. Среди осложнений были: несостоятельность гастростомы с перитонитом (1), пневмоперитонеум (1), кровотечение из брюшной стенки (1), инфильтрат мягких тканей передней брюшной стенки (1), миграция бампера гастростомы в стенку желудка (1).

Несостоятельность гастростомы с перитонитом возникла на 1-е сутки после операции. Лапароскопически выявлено вытекание содержимого желудка в брюшную полость через гастростомическое отверстие, которое было на 0,4 см больше диаметра гастростомической трубки (puc. 2). Осуществлена конверсия в лапаротомию, выполнена реконструкция гастростомы по Кадеру. Пациент выздоровел. 
Пневмоперитонеум наблюдали на 2-е сутки после гастростомии. Проведена компьютерная томография (КТ), выявлен диастаз между передней стенкой желудка и передней брюшной стенкой (рuс.3). Выполнена диагностическая лапароскопия. Вытекания жидкого содержимого желудка в брюшную полость не выявлено. Уменышен свободный ход гастростомической трубки.

Массивное кровотечение из кожной раны, возникшее на 2-е сутки, устранено прошиванием раны.

Инфильтрат передней брюшной стенки, выявленный на 5-е сутки, излечен консервативно в течение 5 дней с применением местных и системных антибиотиков.

Бампер гастростомы мигрировал в стенку желудка у пациента, у которого гастростома находилась 1,5 года без замены. Гастростомическая трубка удалена и рядом установлена новая.

\section{Обсуждение}

ЧЭГ была выполнена впервые в 1980 г. С тех пор операция получила всемирное признание в качестве безопасной техники для обеспечения энтерального питания у больных с нарушениями перорального приема пищи как альтернатива хирургической гастростомии.

Перед выполнением ЧЭГ должна быть четко аргументирована медицинская необходимость и обоснована процедура, учтены абсолютные и относительные противопоказания $[1,3,4]$. При наличии медицинских показаний возрастных ограничений или связанных с массой тела для выполнения гастростомии нет [15 - 17].

Несмотря на то что гастростомия считается безопасной процедурой, она может сопровождаться осложнениями, которые разделяют на малые и большие, связанные с погрешностями в ее техническом выполнении, неправильным уходом за гастростомой $[4,6,18]$. В литературе описаны следующие осложнения ЧЭГ: гнойно-воспалительные изменения мягких тканей передней брюшной стенки в месте наложения гастростомы (инфильтрат, нагноение и др.), парастомическая утечка, некротический фасциит, кровотечение, перфорация желудка, перитонит, кишечная непроходимость, аспирационная пневмония, пневмоперитонеум, повреждение кишечника или печени во время пункции желудка, повреждение слизистой оболочки ротоглотки, гортани, пищевода и желудка при выполнении гастростомии, эрозивно-язвенные изменения слизистой оболочки желудка в месте стомического отверстия и на противоположной стенке желудка, метастатические отсевы по ходу гастростомического канала и на переднюю брюшную стенку (при раке пищевода, ротоглотки), обтурация гастростомической трубки пищей, миграция бампера гастростомы в дистальные отделы пищеварительного канала с кишечной непроходимостью, бампер-синдром (миграция бампера гастростомы в стенку желудка), преждевременное удаление гастростомы, повреждение/разрушение гастростомической трубки [4, 6, 18].

Частота осложнений по результатам нашего исследования составила 4,8\% (95\% Ди 1,6 - 10,8), что сопоставимо с данными, полученными разными авторами - от 3,7 до 35\% [1, 4 - 6, 18]. Мы объясняем это тщательным отбором и подготовкой пациентов к процедуре, строгим со- блюдением технических приемов ее выполнения, адекватным ведением послеоперационного периода.

\section{Выводы}

1. ЧЭГ - безопасный, доступный миниинвазивный метод, который по своим характеристикам превосходит традиционные хирургические методы гастростомии и является методом выбора у пациентов, требующих длительного энтерального питания или декомпрессии желудка при невозможности перорального кормления.

2. Частота осложнений на 105 процедур составила 4,8\% (95\% ДИ 1,6 - 10,8), а в сроки наблюдения 30 дней летальности не было, что обусловлено строгим соблюдением всех технических этапов гастростомии, показаний и противопоказаний, правильным послеоперационным уходом за стомой.

\section{References}

1. Belevich VL, Strukov EJu, Brednev AO, Ovchinnikov DV. Chreskozhnaja jendoskopicheskaja gastrostomija - metod vybora dlja dlitel'nogo jenteral'nogo pitanija. Novosti hirurgii. 2014; 22(6): 750-4. Available from: https://cyberleninka.ru/article/n/chreskozhnaya-endoskopicheskaya-gastrostomiya-metod-vybora-dlya-dlitelnogo-enteralnogo-pitaniya . [In Russian].

2. Deitch EA, Winterton J, Li M, Berg R. The gut as a portal of entry for bacteremia. Role of protein malnutrition. Ann Surg. 1987;205:681-92. Available from: https://www.ncbi.nlm.nih.gov/pmc/articles/PMC1493085/

3. Samigullin MF, Bilalov IV, Duglav EA, Kosolapova TV, Kunduhova FT, Habibulina AR. Chreskozhnaja jendoskopicheskaja mikrogastrostomija kak metod dlitel'nogo jenteral'nogo i lechebno-terapevticheskogo obespechenija pacientov. Kazanskij medicinskij zhurnal. 2017; 98(4): 645-9. Available from: https://journals.eco-vector.com/kazanmedj/article/download/6891/5530. [In Russian]. doi: 10.17750/KMJ2017-645.

4. Rahnemai-Azar AA, Rahnemaiazar AA, Naghshizadian R, Kurtz A, Farkas DT. Percutaneous endoscopic gastrostomy: indications, technique, complications and management. World J Gastroenterol. 2014 Jun 28;20(24):7739-51. Available from: https://www.ncbi.nlm.nih.gov/pmc/ articles/PMC4069302/ doi: 10.3748/wjg.v20.i24.7739.

5. Gomes CA Jr, Andriolo RB, Bennett C, Lustosa SA, Matos D, Waisberg DR, Waisberg J. Percutaneous endoscopic gastrostomy versus nasogastric tube feeding for adults with swallowing disturbances. Cochrane Database Syst Rev. 2015 May 22;(5):CD008096. doi: 10.1002/14651858.CD008096.pub4.

6. Blomberg J, Lagergren J, Martin L, Mattsson F, Lagergren P. Complications after percutaneous endoscopic gastrostomy in a prospective study. Scand J Gastroenterol. 2012 Jun;47(6):737-42. doi: 10.3109/00365521.2012.654404.

7. Rimon E, Kagansky N, Levy S. Percutaneous endoscopic gastrostomy; evidence of different prognosis in various patient subgroups. Age Ageing. 2005 Jul;34(4):353-7. doi: 10.1093/ageing/afi085

8. Bannerman E, Pendlebury J, Phillips F, Ghosh S. A cross-sectional and longitudinal study of health-related quality of life after percutaneous gastrostomy. Eur J Gastroenterol Hepatol. 2000;12(10):1101-9.

9. Gauderer MW, Ponsky JL, Izant RJ. Gastrostomy without laparotomy: A percutaneous endoscopic technique. J Pediatr Surg. 1980;15(6):8725. doi: 10.1016/S0022-3468(80)80296-X.

10. Grant JP. Comparison of percutaneous endoscopic gastrostomy with Stamm gastrostomy. Ann Surg. 1988 May;207(5):598-603. Available from: https://www.ncbi.nlm.nih.gov/pmc/articles/PMC1493508/.

11. Jafri NS, Mahid SS, Minor KS, Idstein SR, Hornung CA, Galandiuk S. Meta-analysis: antibiotic prophylaxis to prevent peristomal infection following percutaneous endoscopic gastrostomy. Aliment Pharmacol Ther. 2007 Mar 15;25(6):647-56. Available from: https://onlinelibrary.wiley.com/doi/abs/10.1111/j.1365-2036.2007.03247.x doi: 10.1111/j.1365-2036.2007.03247.x.

12. Lipp A, Lusardi G. Systemic antimicrobial prophylaxis for percutaneous endoscopic gastrostomy. Cochrane Database Syst Rev. 2013 Nov 14;(11):CD005571. doi: 10.1002/14651858.CD005571.pub3. 
13. Vyawahare MA, Shirodkar M, Gharat A, Patil P, Mehta S, Mohandas KM. A comparative observational study of early versus delayed feeding after percutaneous endoscopic gastrostomy. Indian J Gastroenterol. 2013 Nov;32(6):366-8. doi: 10.1007/s12664-013-0348-8.

14. Islek A, Sayar E, Yilmaz A, Artan R. Percutaneous endoscopic gastrostomy in children: is early feeding safe. J Pediatr Gastroenterol Nutr. 2013 Nov;57(5):659-62.

15. Bochicchio GV, Guzzo JL, Scalea TM. Percutaneous endoscopic gastrostomy in the supermorbidly obese patient. JSLS. 2006 OctDec;10(4):409-13. Available from: https://www.ncbi.nlm.nih.gov/pmc/ articles/PMC3015743/.
16. Senadhi V, Chaudhary J, Dutta S. Percutaneous endoscopic gastrostomy placement during pregnancy in the critical care setting. Endoscopy. 2010;42 Suppl 2:E358-9. doi: 10.1055/s-0030-1256052.

17. Horiuchi A, Nakayama Y, Tanaka N. Transgastric Diversion of Transnasal Long Tube Placement Using a Percutaneous Endoscopic Gastrostomy Site in a Patient with Bowel Obstruction and Massive Ascites due to Ovarian Carcinoma. Case Rep Gastroenterol. 2008 Sep 30;2(3):326-9. Available from: https://www.ncbi.nlm.nih.gov/pmc/articles/PMC3075192/ doi: 10.1159/000155149.

18. Pars H, Çavuşoğlu H. A Literature Review of Percutaneous Endoscopic Gastrostomy: Dealing With Complications. Gastroenterol Nurs. 2017 Dec 6. doi: 10.1097/SGA.0000000000000320. 